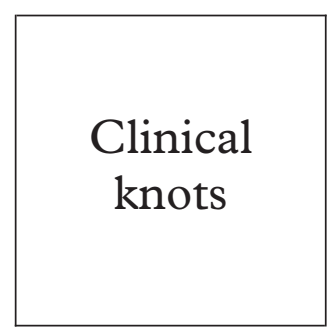

\section{What to do with the patient with recurrent vulvovaginal candidiasis}

\author{
J S Bingham
}

Department of

Genitourinary and

HIV Medicine, Guy's and St Thomas's

Hospitals, London J S Bingham

Accepted for publication 24 May 1999

\section{The problem}

Genital infection with candida species is common in women, $75 \%$ of whom will have had at least one episode during their lives. Indeed, there is a $15-20 \%$ point prevalence of asymptomatic candida carriage in young, nonpregnant, premenopausal women. While acute episodes of candidal vulvovaginitis usually respond rapidly to treatment, some women suffer frequent recurrences or chronic infection and, usually, no predisposing factors can be identified.

Recurrent vulvovaginal candidiasis (RVVC) may be defined as four attacks of mycologically proved candidiasis within 12 months. With asymptomatic carriage there are only small numbers of yeasts $\left(<10^{-3} / \mathrm{ml}\right)$, usually in the blastospore (budding) form. Transition to the hyphal form is associated with symptomatic disease. Although an individual may be culture negative after treatment, yeasts may not be totally eliminated and "vaginal relapse" may occur, usually with the same strain.

It is important, however, to be certain that symptoms are caused by candida; a vaginal swab may be negative but candida can be found on the vulva which is the more common site of symptoms. Here candida needs to be distinguished from a raft of dermatological problems including seborrhoeic dermatitis, eczema, lichen sclerosus et atrophicus, hypersensitivity vulvitis, and psoriasis, among others.

\section{Why does candida recur?}

There are a number of recognised predisposing host factors for genital candidiasis. It occurs more frequently, and is more difficult to treat in women who are pregnant, when broad spectrum antibiotics are given to some women, who are taking oestrogen therapy, who have uncontrolled or poorly controlled diabetes mellitus, who have iatrogenic or therapeutic immune suppression, but probably not in those who have HIV infection, at least not in my experience. Sweat/moisture may increase the virulence of $C$ albicans so tight fitting, noncotton underwear may predispose to infection and female hygiene practices including douching and use of sprays, bubble baths, and "panty liners" can trigger hypersensitivity reactions which may predispose to colonisation with Candida organisms.

Zinc and iron deficiency are no longer thought to predispose to recurrent genital yeast colonisation and, although the same strains may be found in the gut, reinfection from that source is not now believed to be a factor. While trauma during sexual activity may predispose to infection, particularly following abstinence, and sexual partners may harbour the same strain of yeast, sexual transmission is not thought to be a significant factor.

Relapsing candidal vaginitis is almost certainly a result of change in the compartmentalised and protective cell mediated host defence mechanisms at the vaginal mucosal level, rather than humoral or innate immunity. Th 1 and Th 2 cell mediated responses correlate with resistance and susceptibility to mucosal candidiasis. Th 1 type reactivity, with the production of IL2, IFN $\gamma$, and IL12 (stimulating macrophages and polymorphonuclear lymphocytes) and mucosal $\operatorname{IgA}$, is the dominant vaginal response and it maintains asymptomatic candidal colonisation. Th 2 reactivity, with the production of IL4-6, IL10, IgG, histamine, and prostaglandin E2 dominates when endogenous and exogenous factors increase the number of $C$ albicans organisms. This response switches off the protective Th 1 response and promotes immediate hypersensitivity reactions. There is a switch from the blastospore to the hyphal phase and epithelial invasion occurs.

\section{Which organisms cause the problem?}

$C$ albicans is the predominant organism. Other yeasts that may be involved are listed in table 1 . $C$ albicans is almost never azole resistant in HIV negative patients but $C$ glabrata is becoming increasingly prevalent in some populations with RVVC in North America. $C$ glabrata is a budding yeast, without hyphae, and resistance is much more likely to develop during treatment. It is more prevalent in diabetics and there may be an association with the concomitant presence of bacterial vaginosis and with douching. Vaginal discharge is not a major feature and burning, rather than itching of the vulva, may be the main symptom. C krusei is intrinsically resistant to fluconazole but responds to other agents and resistance may also be found with Saccharomyces cerevisiae.

\section{What to do (see fig 1)}

- It is essential, first, to establish the definitive diagnosis. This will involve examination of the patient, screening for genital infections,

Table 1 Yeasts associated with recurrent vulvovaginal candidiasis

Candida albicans, 85-90\%

Candida (Torulopsis) glabrata, 5-10\%

Candida tropicalis, 3-5\%

Candida parapsilosis, 3-5\%

Candida krusei, 1-3\%

Candida kefyr

Candida guilliermondii

Saccharomyces cerevisiae 


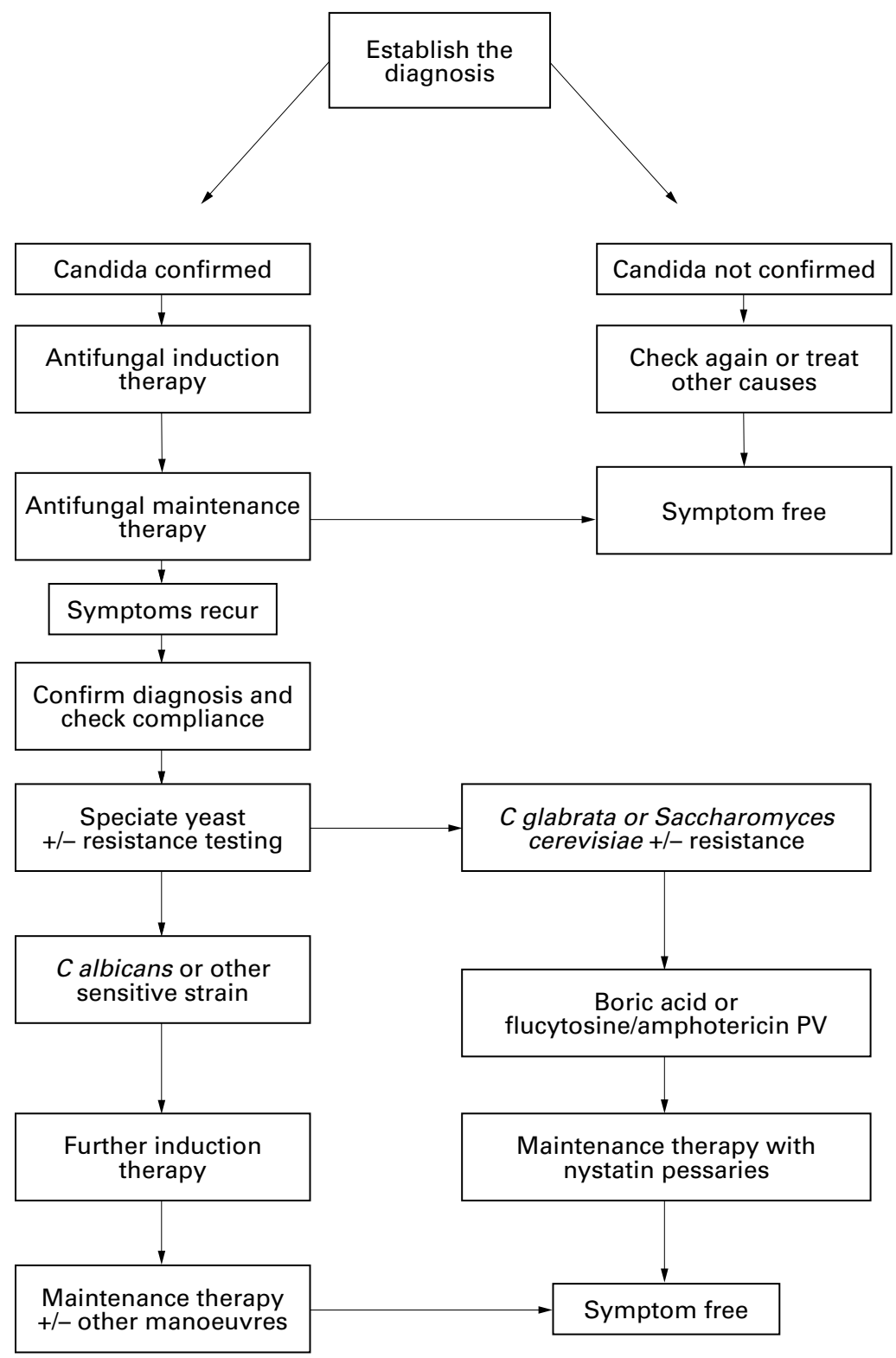

Figure 1 Recurrent vulvovaginal candidiasis.

including looking for yeasts on a Gram stained vaginal smear or on a wet preparation. Culture for yeasts using a transport medium or plating straight onto Sabouraud's medium is helpful, particularly if sensitivity testing is to be carried out, and it is more sensitive than microscopy. Candida may predominate on the vulva and not in the vagina and remember, also, to exclude the other vulval problems mentioned earlier.

- Identify any of the predisposing causes discussed above and manage appropriately. Women who find that a course of antibiotics predisposes to recurrence should be given antifungal cover, either during or at the end of the therapy.

- Once the diagnosis of RVVC is confirmed, induction therapy with imidazoles/triazoles should be prescribed. This may be delivered via the vagina or orally. Some women prefer oral treatment but single dose regimens such as fluconazole $150 \mathrm{mg}$ by mouth immediately are not always effective in these difficult cases and a 1 week course of fluconazole, itraconazole, or ketoconazole may be used. Because of its pharmacokinetic properties, fluconazole need only be given at a dose of $150 \mathrm{mg}$ every third day for three doses. Clinicians should use whichever topical imidazole therapy they prefer for at least 7-14 days; some women find intravaginal cream more satisfactory and soothing than pessaries but always remember to apply cream to the vulva, which is often the main seat of the symptoms.

- Once the symptoms are brought under control the aim is to try to prevent recurrence by the use of maintenance therapy with azole antimycotics. This was first described in 1978 by Davidson and Mould using intravaginal miconazole between the 5 th and 11 th days of the menstrual cycle. Again, therapy can be delivered topically using, for example, clotrimazole $500 \mathrm{mg}$ via the vagina weekly or orally with, for example, fluconazole 100-150 mg immediately by mouth weekly. Itraconazole may also be used. It is usual to continue maintenance therapy for at least 6 months.

- If, despite maintenance therapy, breakthrough occurs or treatment fails, the diagnosis must be confirmed mycologically. If compliance is confirmed then the possibility of azole resistance should be considered. Not all laboratories have facilities for sensitivity testing; if your local laboratory cannot do this for you, you could ask them to send the culture to a mycology reference centre (in the United Kingdom at the PHLS laboratory in Bristol), but minimum inhibitory concentrations for yeasts can be difficult to interpret.

- If it is shown that your patient has a resistant yeast (possibly $C$ glabrata or Saccharomyces cerevisiae), then a different approach will be required. Boric acid can be inserted into gelatin vaginal pessaries by the pharmacy or commercially (in the United Kingdom by Martindale Pharmaceuticals). A dose of 600 $\mathrm{mg}$ twice daily for 14 days has been used by Sobel and others with success. Others have used flucytosine and/or amphotericin in cream or KY jelly for vaginal insertion, again made up by the pharmacy. (For details of doses in this situation contact Dr David White at the Birmingham Heartlands Hospital, Hawthorn House, Department of Sexual Medicine, Bordesley Green East, Birmingham, B9 5SS.) If this manoeuvre proves successful then maintenance therapy cannot be with the same agents, since no safety data are available, and therefore treatment with nystatin pessaries 100000 units at night is advocated. Painting of the vulva and vagina with aqueous gentian violet, as an adjunct to other therapies, may be helpful in some difficult cases.

- In really intractable cases other manoeuvres may be tried. There is some evidence that inducing a state of relative oestrogen deficiency by the use of progestogen containing contraceptives can be helpful occasionallyfor example, use of Depo-Provera in those requiring contraception, or cyclical oral medroxyprogesterone or dydrogesterone in 
the second half of the menstrual cycle. Others have advocated the ingestion, or intravaginal insertion of live yoghurt (Lactobacillus acidophilus) and, in theory, desensitisation using a laboratory prepared Candida antigen could have a place in chronic cases.

- Remember the art of medicine; explain the problem to the woman and the rationale of your therapy. Try to reassure her and, where there is a negative impact on her self image or psychological or relationship problems resultant on her condition, arrange suitable counselling. It should be possible to help all patients and cure some.

Based on a contribution given at an Ordinary General Meeting of the Medical Society for the Study of Venereal Diseases on 27 November 1998.

\section{Useful references}

Fidel PL. Vaginal candidiasis: review and role of local mucosal immunity. AIDS Patient Care and STD 1998;12:359-66.

Fidel PL, Sobel JD. Immunopathogenesis of recurrent vulvovaginal candidiasis. Clin Microbiol Rev 1996;9:335-48.

White DJ, Emens M, Shahmanesh M. Recurrent vulvovaginal candidosis. Int F STD AIDS 1991;2:235-9.
White DJ, Johnson EM, Warnock DW. Management of persistent vulvovaginal candidosis due to azole-resistant Candida glabrata. Genitourin Med 1993; 69:112-14.

Sobel JD. Vulvovaginitis - when Candida becomes a problem. Dermatol Clin 1998;16:763-8.

Sobel JD. Pathogenesis and treatment of recurrent vulvovaginal candidiasis. Clin Infect Dis 1992;14:S148-53.

Sobel JD. Controversial aspects in the management of vulvovaginal candidiasis. $\mathcal{F}$ Am Acad Dermatol 1994;31:S10-

Shinohara YT, Tasker SA. Successful use of boric acid to control azole-refractory. Candida vaginitis in a woman with AIDS. $\mathcal{F}$ Acquir Immun Defic Syndr Human Retrovirol 1997;16:219-20. Redondo-Lopez V, Lynch M, Schmitt C, et al. Torulopsis glabrata vaginitis: clinical aspects and susceptibility to antifungal agents. Obstet Gynaecol 1990;76:651-5.

Sobel JD, Chaim W. Treatment of C glabrata vaginitis: retrospective review of boric acid therapy. Clin Infect Dis 1997;24:649-52.

Jovanovic R, Congema E, Nguyen HT. Antifungal agents vs boric acid for treating mycotic vulvovaginitis. $\mathcal{f}$ Reprod Med 1991;36:593-7.

Hilton $\mathrm{E}$, Isenberg $\mathrm{HD}$, Alperstein $\mathrm{P}$, et al. Ingestion of yoghurt containing Lactobacillus acidophilus as prophylaxis for Candida vaginitis. Ann Intern Med 1992;116:353-7.

Drutz DJ. Lactobacillus prophylaxis for Candida vaginitis. Ann Intern Med 1992;116:419-20.

Rigg D, Miller MM, Metzger WJ. Recurrent allergic vulvovaginitis with Candida albicans allergen immunotherapy. $A m \mathcal{F}$ Obstet Gynecol 1990;162:332-6.

Deennerstein GJ. Depo-Provera in the treatment of recurrent vulvovaginal candidiasis. F Reprod Med 1988;31:801-3.

Davidson F, Mould RF. Recurrent genital candidosis in women and the effect of intermittent prophylactic treatment. $\mathrm{Br} F$ Vener Dis 1978;54:176-83. 